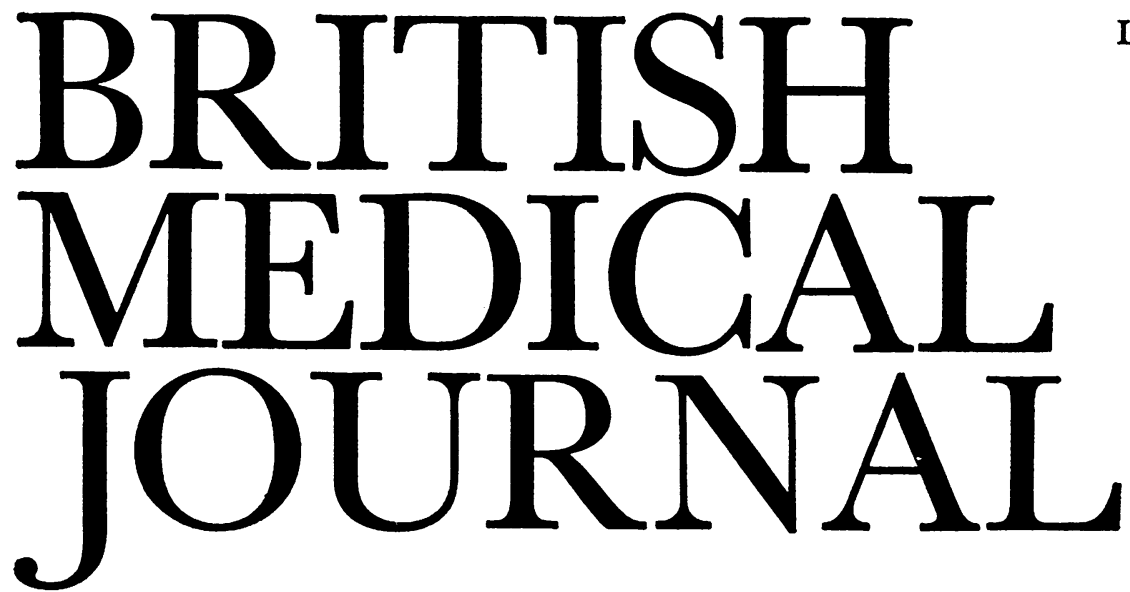

\title{
Cardiac Transplantation Today
}

N. E. Shumway and his colleagues at Stanford University, California, had already gained considerable experience in transplantation of the canine heart ${ }^{1}$ when C. Barnard and his team performed the first operation in man in $1967 .{ }^{2}$ During the next two years over 100 human heart transplants were performed throughout the world, and controversy ran high over the many new ethical, religious, and medicolegal problems that were raised. ${ }^{34}$ Though seemingly the ultimate in cardiac surgery, transplantation is not technically as demanding as, say, operation for neonatal coarctation or a difficult Fallot's tetrad, and for a while transplantation threatened to become a common procedure. It soon became apparent that the operation which Shumway had developed over many years could lead to successful function of the transplanted heart at least initially, 5 but tissue incompatibility leading to progressive damage to the transplant and its rejection seemed likely to prevent long-lasting success, and the many early deaths from rejection were dispiriting. The last two years have seen a welcome restriction of these operations to a small number of centres. While work on the immunological problems has been intensive, there has been a notable absence of recent news of the long-term results of transplantation. A new report from Shumway's group is therefore of exceptional interest. ${ }^{6}$

This report concerns 26 patients who underwent cardiac transplantation at Stanford during 1970. The underlying disorder was coronary artery disease in 21 patients and primary cardiomyopathy in five patients. Twenty-three of the patients were men, and the average age was 50 years. $\mathrm{ABO}$ blood group and lymphocyte compatibility were assured before operation. ${ }^{7}$ Tissue typing uncovered between one and three mismatches in all except two patients, in whom no mismatches were detected. Immunosuppressive therapy with azathioprine, steroids, and antilymphocytic globulin was given to all patients, and acute rejection episodes were treated with intravenous methyl prednisolone, actinomycin $\mathrm{D}$, and heparin.

Thirteen of the patients died in hospital. Four deaths were due to infection and three to acute rejection; three were attributed to right ventricular failure secondary to pulmonary hypertension. Thirteen patients left the hospital, of whom seven have so far survived beyond one year. Ten patients have now been fully appraised by rightand left-heart catheterization, and the results are important.

All the right-side pressures were within the normal range at rest. The left ventricular end-diastolic pressures were near normal at rest and the cardiac outputs were in the low normal or subnormal range. Some tests were carried out to detect reinnervation of the hearts. Carotid massage, Valsalva manoeuvre, amyl nitrite, and atropine were followed by appropriate rate responses from the recipient atrial remnant which was monitored by an intra-atrial electrode catheter, but no significant rate changes occurred in the donor heart. It was concluded that the transplanted heart had in each case remained totally without nerve supply. Nevertheless the output of the transplanted heart rose steadily on exercise on a line parallel to but below that expected for a normal heart.

During the first three minutes of exercise the heart rate increased only modestly, but the left ventricular enddiastolic pressures went up. This rise was associated with an increase in stroke volume, which largely accounted for the early rise in output. With further exercise the stroke volume remained stable at the higher figure but the heart rate increased further. The early rise in stroke volume was thought to be probably due to the Frank Starling mechanism, whereby an increase in left ventricular volume is followed by an increase in the force of contraction. This is a fundamental property of normal myocardium and is independent of nerve supply, but the phenomenon may not be demonstrable in diseased hearts. The late increase in rate was attributable to medullary release of catecholamine, to which the heart, if denervated, would be unduly sensitive.

Both mechanisms occurring simultaneously account for the response of the normal heart to effort. The only difference in the denervated hearts was that the effects were sequential owing to the lack of intracardiac release of catecholamine from nerve endings within the heart, which normally determine the early rate response to exercise. These results are similar in every way to those reported for the denervated dog heart ${ }^{8}$ and for the autotransplanted dog heart. 9

Thus these year-old transplanted human hearts performed astonishingly well. Despite episodes of rejection and the disadvantages of denervation the transplanted hearts performed in a way that was consistent with normal activity beyond one year after transplantation. Moreover $75 \%$ of the patients who were alive two months after transplant were alive after a year, and $60 \%$ of those who left hospital were still alive at the end of two years.

This report is remarkably encouraging and certainly 
justifies the determination of Shumway's group to pursue research in human cardiac tramsplantation. The lessons seem to be clear. The early mortality is high, but these patients were in preterminal heart failure, and a similar mortality figure was reported not long ago for triple valve replacement. 10 We can expect to achieve better control of the immunological problems in future and thus lower the early heavy losses from acute rejection (too little suppression) or from infection (which is often the sequel of too much). Only when these hazards have receded will it be justifiable to operate on patients before they reach the stage of failure of all organs from inadequate perfusion. Then, as with valve replacements, the operation mortality will fall substantially.

Suitable candidates for cardiac transplantation at present are patients with permanent incapacity due either to ischaemic damage or to cardiomyopathy. Moderate pulmonary hypertension is reversible, but severe pulmonary vascular disease may pose too severe a burden on the donor right ventricle. Infants and young people with complex congenital cardiac defects should not be subjected to this operation until the donated heart is known to have a reasonably long expectation of survival and function.

The excellent performance of Shumway's patients after a year and the relatively low later mortality in his survivors are tremendously encouraging. The report from his pioneer group gives hope that cardiac transplantation may yet be more generally undertaken in the major cardiac centres and so provide real palliation in myocardial disease.

\footnotetext{
1 Shumway, N. E., Angell, W. W., and Wuerflein, R. D., Transplantation, $1967,5,900$.

2 Barnard, C. N., American fournal of Cardiology, 1968, 22, 584

Dempster, W. J., Melrose, D. G., and Bentall, H. H., British Medical Fournal, 1967, 1, 177

Goodwin, J. F., and Oakley, C. M., American Heart Fournal, 1969, 77,

437.
Cooley, D. A., Bloodwell, R. D., Hallman, G. L., and Nora, J. J., fournal of the American Medical Association, 1968, 205, 479.

Griepp, R. B., Stinson, E. B., Dong, E., Clark, D. A., and Shumway, N. E., Surgerv, 1971, 70, 88.

T. E., Surgery, 1971, 70, 88.

Botha, M. C., South African Medical fournal

- Donald, D. E., Circulation, 1968, 38, 225. tion, 1967, 35, 96.

10 Kittle, C. F., et al., Circulation, 1969, 39, 169.
}

\section{Compression of Coeliac Axis}

Reports continue to appear on the coeliac axis compression syndrome. Abdominal pain is said to be provoked by partial occlusion of the coeliac axis. There are now some 80 well-documented cases, and the latest paper, from Canada, ${ }^{1}$ is unique in that not only symptoms but gross organic changes are attributed to ischaemia from coeliac axis stenosis. Do all these cases exemplify a real syndrome?

It was first described by J. D. Dunbar and his colleagues ${ }^{2}$ from Columbus, Ohio, in 1965, when 15 cases were presented. This group has the largest reported series to its credit with 30 cases. $^{3}$ If these cases all represent the same syndrome, it has the following features. Eighty-one per cent. are women, and though some reports stress youth as an important criterion ${ }^{34}$ the average age is 43 years; two series show mean ages of $51^{5}$ and 52.6 Symptoms are of upper abdominal pain and bloating immediately after food, ${ }^{4}$ and in others the pain is said to be continuous. ${ }^{1}$
Loss of weight and diarrhoea-two cardinal symptoms of mesenteric ischaemia-are not specially notable and were respectively present in $35 \%$ and $42 \%$ of all reported cases. The patients tended to be leptosomatic and asthenic, but the only abnormal physical sign was an upper epigastric non-radiating bruit. ${ }^{34}$ The diagnosis is established by biplanar angiography, in which the lateral aortogram shows extrinsic anterior narrowing of the coeliac axis with poststenotic dilatation. Enlargement of the pancreatico-duodenal arteries as collaterals between coeliac and superior mesenteric vessels is seen on the anteroposterior films. Selective catheterization of the coeliac axis is impossible, but a superior mesenteric angiogram fills the coeliac axis via collaterals and flow is delayed through its branches. ${ }^{24}$ No other investigation appears to help diagnosis. ${ }^{3}$

But there are obstacles to accepting the validity of the syndrome. The symptoms attributed to it are rather vague and highly subjective, and in some cases the patients have had psychiatric care. ${ }^{19}$ The significance of the only physical sign-the bruit-is not great, ${ }^{4} 10$ and the $x$-ray appearances may be incidental, since they may be seen in asymptomatic people. ${ }^{1} 911$ Objective measurement is difficult or impossible.

The factors concerned in visceral ischaemia are comolex. The blood supply to the gut must be rich enough to sustain the high cellular turnover of the intestinal mucosa and in addition carry sufficient reserve to meet the enormous requirements of digestion. Moreover, the visceral circulation is peculiarly dependent on an adequate arterial inflow, for it has to contend with the raised venous outflow pressure of the portal tract and yet is devoid of barorecentors necessary for local protective vasomotor reflexes. ${ }^{12}$ However, there is a huge collateral potential between the three maior arteries to the gut-namely, coeliac and the superior and inferior mesenteric. In favourable circumstances any one artery can take over the distributive fields of the others. The extent of this capacity was first shown in 186913 in a postmortem study but has been confirmed surgically since. ${ }^{14}$ Indeed L. H. Appleby ${ }^{15}$ has advocated ligature of the coeliac axis for the treatment of stomach cancer and reported 13 cases without any apparent ischaemic problems. It has usually been maintained that two out of three of the visceral arteries have to be occluded before ischaemia of the gut is produced, ${ }^{16-18}$ lesser degrees of obstruction being insignificant. J. R. Derrick and colleagues ${ }^{19}$ found narrowing of the coeliac axis in $44 \%$ of unselected necropsy cases. From angiographic studies A. P. Dick and his co-workers ${ }^{20}$ calculated that the total cross-sectional area of the three arteries to the gut had to be reduced below two-thirds before ischaemia was produced. This sort of evidence has to be discarded if partial occlusion of the coeliac axis is accepted as giving rise to symptomatic ischaemia. However, A. J. Edwards and colleagues ${ }^{4}$ showed that a "steal" or an intermittent hyperaemic flow pattern could occur in a manner that would allow the symptoms to be vascularly determined without negating the above evidence, though they did not favour this hypothesis. In their hands the restoration of the flow pattern to normal was not always accompanied by loss of symptoms. It would seem most unlikely that isolated narrowing of the coeliac artery could, as has been claimed, cause gastric fibrosis, haemorrhagic gastritis, pancreatic insufficiency, ${ }^{1}$ or hepatic cirrhosis. ${ }^{21}$ The coincidence of organic disease and narrowing of the coelic axis does not prove the disease to be due to the constriction and at the same time it prompts the suggestion that the symptom may not be due to the arterial deformity at all. 